EPJ Web of Conferences 110,01007 (2016)

DOI: $10.1051 /$ epjconf/201611001007

(C) Owned by the authors, published by EDP Sciences, 2016

\title{
EVALUATION OF HEAT TRANSFER COEFFICIENTS DURING THE WATER VAPOR CONDENSATION CONTAINED IN THE FLUE GAS
}

\author{
Victor Bespalov ${ }^{1, \text { a }}$, Vladimir Bespalov ${ }^{1}$, Denis Melnikov ${ }^{1}$ \\ ${ }^{1}$ National Research Tomsk Polytechnic University, 634050 Tomsk, Russia
}

\begin{abstract}
Is shown the influence of the heat transfer coefficient from the wet flue gas to the heat exchange surface on the overall heat transfer coefficient in the gas-air heat exchanger with the water vapor condensation. Experimental data are compared with calculations based on the mathematical model of the condensing heat exchanger.
\end{abstract}

\section{Introduction}

Thermal design of the heat exchanger surface is a classical task in heat engineering. But heat transfer from two-component medium when changing the aggregate state one of the components has certain complexity. Such task is necessary to solve when the mathematical model for flue gas heat recovery condenser is developing. [1]. Installation scheme is shown in Figure 1.

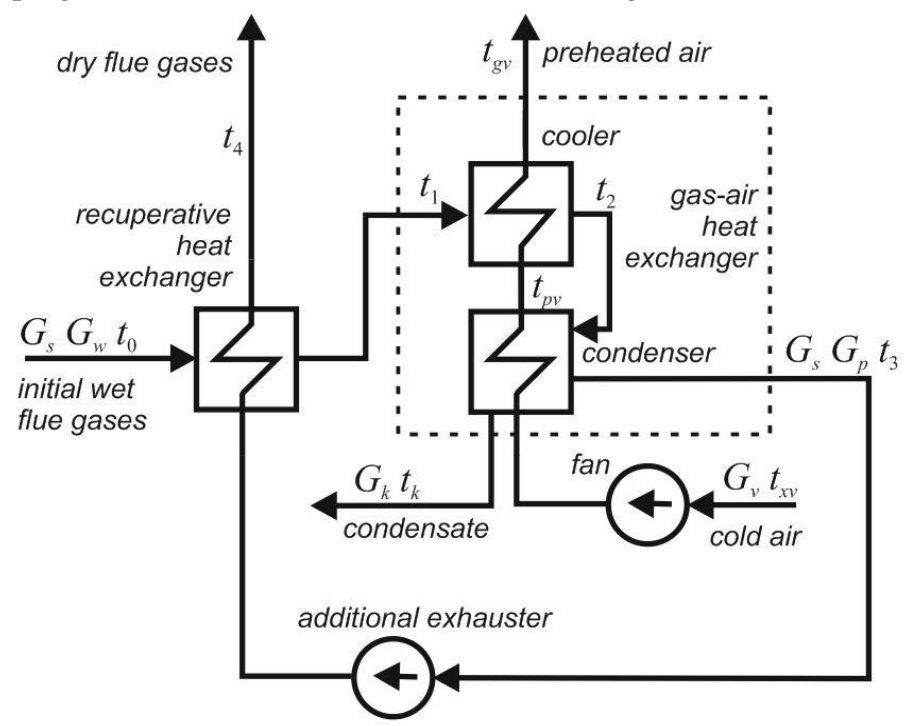

Figure 1. Deep heat recovery of flue gas, installation scheme.

\footnotetext{
${ }^{\mathrm{a}}$ Corresponding author: vic@tpu.ru
} 
Initial flue gases are pre-cooled in the recuperative gas-gas surface plate-type heat exchanger by heating the dry flue gases. Then they are fed to the gas-air surface plate-type heat exchanger. Structurally, it is a single unit conventionally divided by two parts: cooler and condenser. This heat exchanger heats the cold air supplied by the fan. The heating carried out by cooling the flue gases and using the latent heat of water vapor condensation. Drained flue gases for heating are fed to a heat exchanger by means of the additional exhauster to compensate aerodynamic resistance into the flues, heat exchanger and condenser.

\section{Research methodologies}

The mathematical model of the installation includes the heat and material balance equation, as well as heat and mass transfer equations. The input variables are the cold air temperature $t_{x v}$ and the initial flue gas mass flow with initial temperature $t_{0}$, which consist of a dry flue gas $G_{s}$ and water vapor $G_{w}$. Heat exchange surface area can be calculated if set in the first approximation the heat transfer coefficients of each heat exchanger. Based on the calculated heat exchange surface areas the heat exchangers can be designed. The geometrical parameters of heat exchange surface areas allow to clarify the heat exchangers heat transfer coefficients and to adjust the streams temperature. The heat transfer coefficient is calculated as follows [2]:

$$
k_{t}=\frac{1}{\frac{1}{\alpha_{1}}+\frac{\delta_{s}}{\lambda_{s}}+\frac{1}{\alpha_{2}}},
$$

where $\alpha_{1}$-coefficient of heat transfer from the hot medium (flue gases) to the heat exchange surface, $\delta_{s}$ - sheet thickness, $\lambda_{s}$ - thermal conductivity of sheet, $\alpha_{2}$ - coefficient of heat transfer from the heat exchange surface to the heated medium.

For heat exchangers where both gaseous media do not have a phase transition (no water vapor condensation), the heat transfer coefficients are calculated by the usual method and depend mainly on the gas velocity. This is true for calculation the heat transfer coefficient of the recuperative heat exchanger and the cooler. When calculating the condenser heat transfer coefficient the coefficient of heat transfer from the heat exchange surface to the air flow is calculated in the same way. For air flow under atmospheric pressure criterial equation is

$$
\mathrm{Nu}=0,018 \cdot \mathrm{Re}^{0,8},
$$

where $\operatorname{Re}=w \cdot D / v, w$-air velocity, $D$ - equivalent diameter of the channel and $v$-air kinematic viscosity. The coefficient of heat transfer from the heat exchange surface to the air flow is

$$
\alpha_{2}=\mathrm{Nu} \cdot \lambda / D
$$

Air velocity is limited to a quadratic increasing aerodynamic drag of the air tract. When the air velocity above $18 \mathrm{~m} / \mathrm{s}$ the heat exchanger resistance exceeds $1 \mathrm{kPa}$. It is undesirable due to the fan characteristics to supply air. Turbulent airflow begins at speeds of over $3 \mathrm{~m} / \mathrm{s}$. For an acceptable heat transfer the air velocity must be $13 \div 18 \mathrm{~m} / \mathrm{s}$, and the heat transfer coefficient is $57 \div 75 \mathrm{~W} /\left(\mathrm{m}^{2} \cdot \mathrm{K}\right)$.

The main difficulty is the determination of the heat transfer coefficient from the wet flue gas to the heat transfer plate surface $\alpha_{1}$ during the water vapor condensation contained in them. Proceeding from the relation (1), with known thermal resistance of the heat transfer surface and the heat transfer coefficient from the surface to the air flow $\alpha_{2}$, it is possible to assess the impact $\alpha_{1}$ to overall heat transfer coefficient $k_{t}$ (Figure 2). 


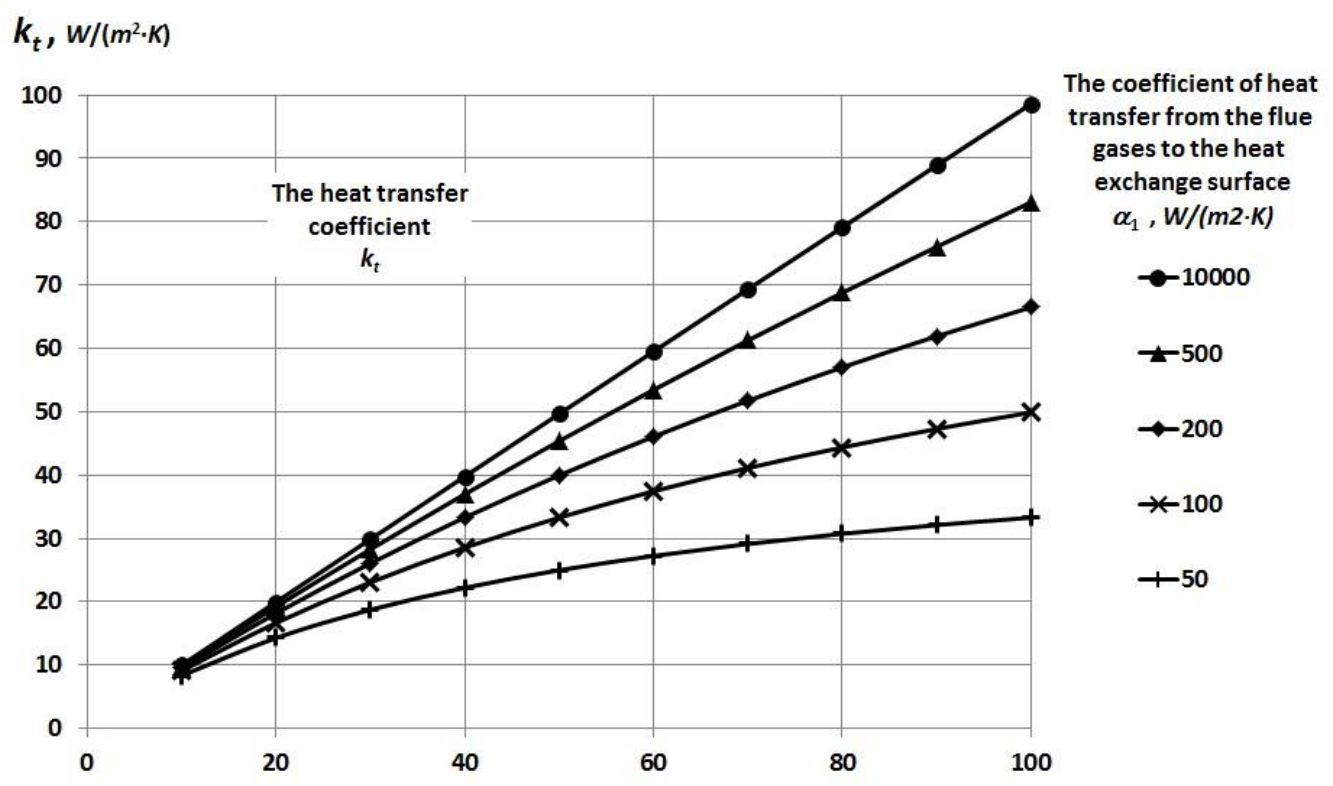

The coefficient of heat transfer from the heat exchange surface to the air $\alpha_{2}, W /(m 2 \cdot K)$

Figure 2. The $\alpha_{1}$ influence on the heat transfer coefficient.

For small values $\alpha_{1}\left(\alpha_{1}<<\alpha_{2}\right)$ the heat transfer coefficient depends mainly on the heat transfer from the condensable vapor in the flue gas stream $\alpha_{1}$. For large values $\alpha_{1}\left(\alpha_{1}>>\alpha_{2}, \alpha_{1}>500\right)$, the heat transfer coefficient to a greater extent depends on the heat transfer from the air $\alpha_{2}$. In the considered heat recovery condenser $\alpha_{2}$ less than $100 \mathrm{~W} /\left(\mathrm{m}^{2} \cdot \mathrm{K}\right)$. Due to condensation, the heat transfer from the wet flue gas is much higher if turbulent regime is provided.

For today is not developed a universal method of calculating the heat transfer coefficient from the flue gas (or vapor mixture at low vapor content) with water vapor condensation to the heat transfer surface. All known methods are based on the experimental data results analysis and offer the equations in rather narrow range of heating media physical parameters. Choosing and applying the calculation method for functionally similar heat recovery units requires analysis and justification. Simulation of the process carried out by the Moscow Energy Institute scientists, led by professors Y. Kuzma-Kichta, A. Sedlov, A. Solodov, A. Garjaev [3, 4, 5]. In the thesis D. Buhonov gives an overview and analysis the known calculation methods of condensing heat exchange systems and their applicability to the heat recovery units calculation. Deviations of heat transfer coefficients according to known techniques are $70 \div 500 \%$. These authors offer its own method for calculating the heat transfer coefficient from steam-air mixture to the heat transfer surface [6]. Another technique is described by Professor A. Kudinov from Samara State Technical University [7, 8, 9]. This technique is based on the experimental data analysis by testing the operated heat recovery units. Corresponding coefficients were calculated for the criterial equation derived by similarity principle. The same methods apply by other authors $[10,11]$.

Calculated by these methods, heat transfer coefficients from vapor condensed flue gases to the surface of described above heat recovery condenser showed a very large difference (from 52 to 800 $\left.W /\left(m^{2} \cdot K\right)\right)$.

\section{Results}

In Tomsk CHP-3 was created an experimental installation for flue gas deep heat recovery. Five experiments were performed, each lasting 30 to 50 minutes, to determine the experienced heat transfer 
coefficients. The error in determining the heat transfer coefficient in this experiment series is within $15 \%$. Table 1 summarizes the processing experiment results compared with the results calculated by mathematical model of heat transfer process in a condenser. The input data for the mathematical model were the experimental parameters and the geometric characteristics of the experimental installation.

Table 1. Comparing the experimental data with calculated by the mathematical model.

\begin{tabular}{|c|c|c|}
\hline Parameter & $\begin{array}{l}\text { The average } \\
\text { values by } \\
\text { experiments }\end{array}$ & $\begin{array}{c}\text { Calculated } \\
\text { values by the } \\
\text { mathematical } \\
\text { model }\end{array}$ \\
\hline Excess air ratio $\alpha$ & 2,1 & 2,1 \\
\hline The average air temperature entering the condenser $t_{x v},{ }^{\circ} \mathrm{C}$ & 3,8 & 3,8 \\
\hline The average air temperature at the condenser outlet $t_{g v},{ }^{\circ} \mathrm{C}$ & 29,7 & 25,1 \\
\hline The average wet flue gas temperature entering the condenser $t_{1},{ }^{\circ} \mathrm{C}$ & 56,5 & 56,5 \\
\hline The average dry flue gas temperature at the condenser outlet $t_{3},{ }^{\circ} \mathrm{C}$ & 37 & 35,8 \\
\hline $\begin{array}{l}\text { The moisture content of initial wet flue gases, grams per kilogram of dry } \\
\text { gas, g/kg.d.g. }\end{array}$ & 74,4 & 74,4 \\
\hline The moisture content of dried flue gases, g/kg.d.g. & 36,5 & 38,1 \\
\hline The volumetric flue gas flow rate, $\mathrm{m}^{3} / \mathrm{c}$ & 0,108 & 0,100 \\
\hline The theoretical flow rate of condensate, $\mathrm{kg} / \mathrm{s}$ & 0,0046 & 0,0042 \\
\hline The volumetric air flow rate, $\mathrm{m}^{3} / \mathrm{c}$ & 0,493 & 0,4900 \\
\hline The thermal power transfered to the air from the flue gas $Q, k W$ & 13,00 & 13,00 \\
\hline The average temperature difference $\Delta t,{ }^{\circ} \mathrm{C}$ & 23,0 & 26,34 \\
\hline The overall heat transfer coefficient $k, W /\left(m^{2} \cdot K\right)$ & 61,8 & 67,30 \\
\hline The air velocity in the condenser cross section $w, m / c$ & 22,35 & 19,33 \\
\hline $\begin{array}{l}\text { The heat transfer coefficient from the heat transfer surface to the air } \alpha_{2} \text {, } \\
W /\left(m^{2} \cdot K\right)\end{array}$ & 82 & 73,70 \\
\hline $\begin{array}{l}\text { The heat transfer coefficient from wet flue gases to the heat transfer surface } \\
\alpha_{1}, W /\left(m^{2} \cdot K\right)\end{array}$ & 251 & 798,1 \\
\hline
\end{tabular}

As can be seen from the experimental results, the heat transfer coefficient $k=62 \pm 6 \mathrm{~W} /\left(\mathrm{m}^{2} \cdot \mathrm{K}\right)$. The calculated heat transfer coefficient from the heat transfer surface to the air $\alpha_{2}=82 \pm 4 \mathrm{~W} /\left(\mathrm{m}^{2} \cdot \mathrm{K}\right)$ and a known plate thermal resistance allow to determine the heat transfer coefficient from the wet flue gases to the heat exchange surface by the equation (1). The last one considering the error was found to equal $\alpha_{1}=251 \pm 50 \mathrm{~W} /\left(\mathrm{m}^{2} \cdot \mathrm{K}\right)$.

A mathematical model of heat transfer in the condenser is built on determining the overall heat transfer coefficient by calculating the heat transfer from the wet flue gases to the sheet cooled by air on the other side. The heat transfer coefficient $\alpha_{1}$ calculated by Kuzma-Kichta equations in the mathematical model gives a result $\alpha_{1}=798 \mathrm{~W} /\left(\mathrm{m}^{2} \cdot \mathrm{K}\right)$. 


\section{Conclusion}

- A determining influence on the overall heat transfer coefficient in the gas-air heat exchanger with heating air and water vapor condensation in the flue gases has the heat transfer coefficient from the sheet to the air $\alpha_{2}$. When air velocity comparable to flue gas the heat transfer coefficient from them to the sheet is higher $\alpha_{1}>\alpha_{2}$ due to heat exchange intensification by the water vapor condensation.

- Existing methods of calculating the heat transfer coefficient from the wet flue gases to the heat exchange surface with the water vapor condensation do not allow to obtain results that would provide a good agreement with experimental results. Should be carried theoretical and experimental study of heat transfer between the flue gas (or gas mixture) and the cooling surface, when contained therein water vapor are condensing.

\section{References}

1. V. Bespalov, Power engineering of Tatarstan, 38, 32 (2015)

2. T. Bergman, F. Incropera, A. Lavine, Fundamentals of Heat and Mass Transfer (USA, John Wiley \& Sons, 2011)

3. A. Garjaev, E. Tseplyaeva, Sur l'efficacite energetique, 1, 187 (2003)

4. A. Garyaev, Thermal Engineering, 52, 573 (2005)

5. N. Dudnik, A. Garyaev, Thermal Engineering, 57, 522 (2010)

6. Y. Kuzma-Kichta, D. Bukhonov, Y. Borisov, Thermal Engineering, 54, 210 (2007)

7. A. Kudinov, M. Kalmykov, Thermal Engineering, 49, 685 (2002)

8. S. Ziganshina, A. Kudinov, Power Technology and Engineering, 44, 231 (2010)

9. A. Kudinov, V. Antonov A., Y. Alekseev, Thermal Engineering, 47, 65 (2000)

10. X. Shi, D. Che, B. Agnew, J. Gao, International Journal of Heat and Mass Transfer, 54, 606 (2011)

11. K. Jeong, M. Kessen, H. Bilirgen, E. Levy, International Journal of Heat and Mass Transfer, 53, $2361(2010)$ 